\title{
Subclavian steal syndrome as the presenting feature of hypervascular thyroid nodule
}

\author{
M. Kanko, E. Ciftci ${ }^{1}$, H. Efendi ${ }^{2}$, K. T. Berki \\ Departments of Cardiovascular Surgery, ${ }^{1}$ Radiodiagnostic and ${ }^{2}$ Neurology, Kocaeli University, Kocaeli, Turkey
}

\begin{abstract}
Subclavian steal syndrom (SSS) is a clinical entity characterized by brachial and basilar insufficiency as a result of critical proximal subclavian artery stenosis or occlusion. We report a patient of giant hypervascular thyroid nodule presenting with features of SSS. The left hand ischemia and symptoms of vertebro-basilar artery in our patient were probably related to stealing of blood by the hypervascular thyroid nodule from the subclavian artery. The patient was relieved of the symptoms upon percutaneous subclavian stent placement
\end{abstract}

Key words: Subclavian steal syndrome, stent, thyroid adenoma.

Subclavian steal syndrome (SSS) is caused by a hemodynamically critical proximal subclavian artery stenosis or occlusion with subsequent retrograde filling of the subclavian artery via the contralateral vertebral artery. The stenosis causes a decrease in the blood flow to the brain and ipsilateral upper extremity on the affected side, resulting in vertebro-basilar insufficiency and ischemia of the affected extremity. ${ }^{[1-3]}$ We report a patient of giant hypervascular thyroid nodule presenting with features of SSS.

\section{Case Report}

A 41-year old woman was admitted for evaluation of headache, dizziness and left hand ischemic changes. She used to experience left upper limb weakness, claudication and syncopal attacks on physical activity of left upper limb. About 10 days before admission she used to experience intermittent color changes of the skin in the affected limb which later become a permanent feature. She was diagnosed as a case of schizophrenia and was on regular medication. Examination revealed absent left radial pulse and a murmur over left subclavian artery (LSA) region. There was difference in blood pressure $(>50 \mathrm{~mm} \mathrm{Hg}$ ) between the two upper limbs. Diagnostic workup was negative for connective tissue diseases, vasculitis and vasospastic diseases. Color Doppler sonography of the LSA demonstrated a dampened waveform with low systolic flow velocities and loss of diastolic backflow. Left vertebral artery (LVA) sonography showed incomplete SSS. In the provocation test, the blood flow in the LVA was below baseline values. Ultrasonography revealed a hypervascular nodule $4 \mathrm{~cm}$ in diameter in the left lobe of the thyroid gland. Thyroid scintigraphy revealed a hot thyroid nodule. Thyroid hormonal profile was normal. Fine needle aspiration cytology inconclusive. Diagnostic angiography revealed 70\% ostial LSA stenosis and a hypervascular lesion in the left lobe of the thyroid gland [Figures 1-3]. Contralateral subclavian artery injection showed retrograde flow in the LVA supplying the left thyroid nodule and left axillary artery simultaneously. A balloon-expandable stent (LD express 8 x $25 \mathrm{~mm}$, Boston scientific, USA) was deployed across the subclavian artery. Postprocedure angiogram revealed a patent subclavian artery and good axillary artery circulation [Figure 4]. In the follow-up she was relieved of limb ischemic symptoms and signs as well as features of vertebro-basillary insufficiency. The

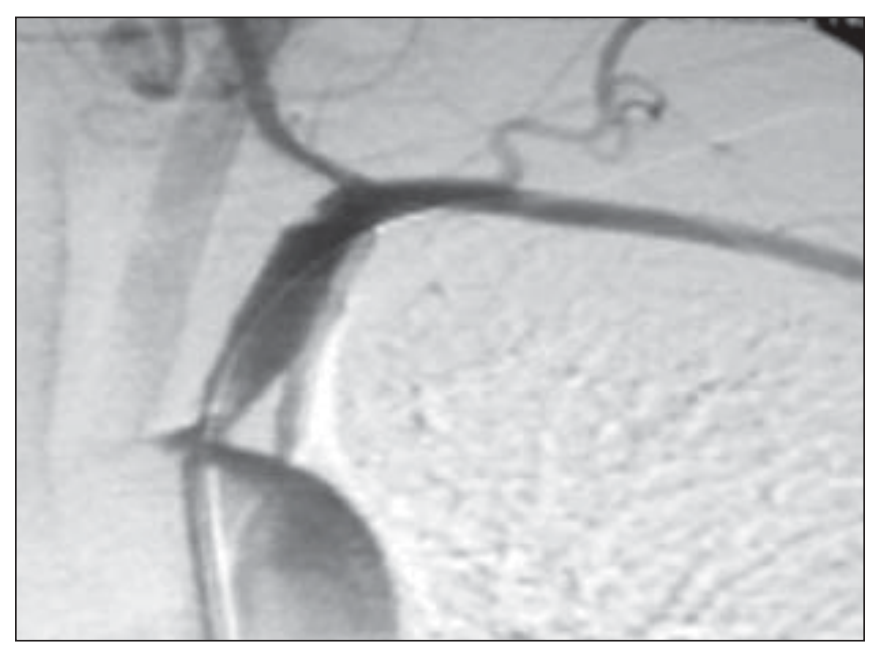

Figure 1: Anteroposterior image of left subclavian artery shows approximately $70 \%$ stenosis 


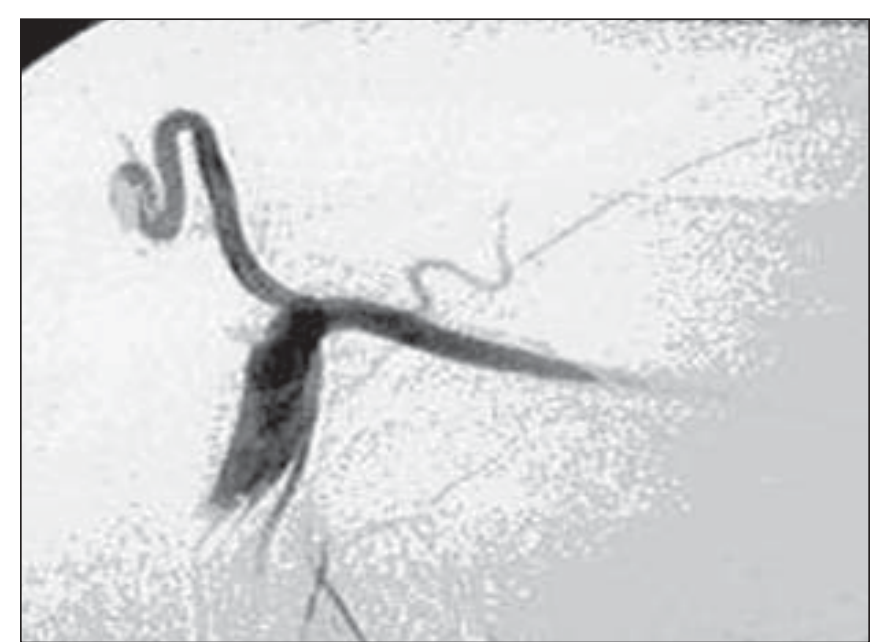

Figure 2: Thyrocervical trunk is filling before axilary artery

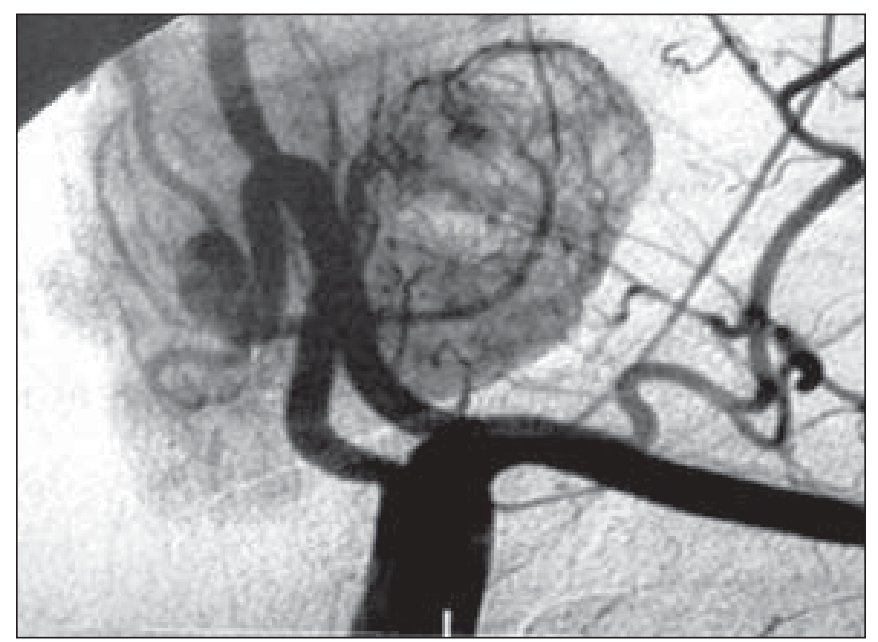

Figure 3: Anteroposterior images of the left subcavian artery and thyrocervical trunk show hypervascular tyroid nodul and normal left vertebral artery

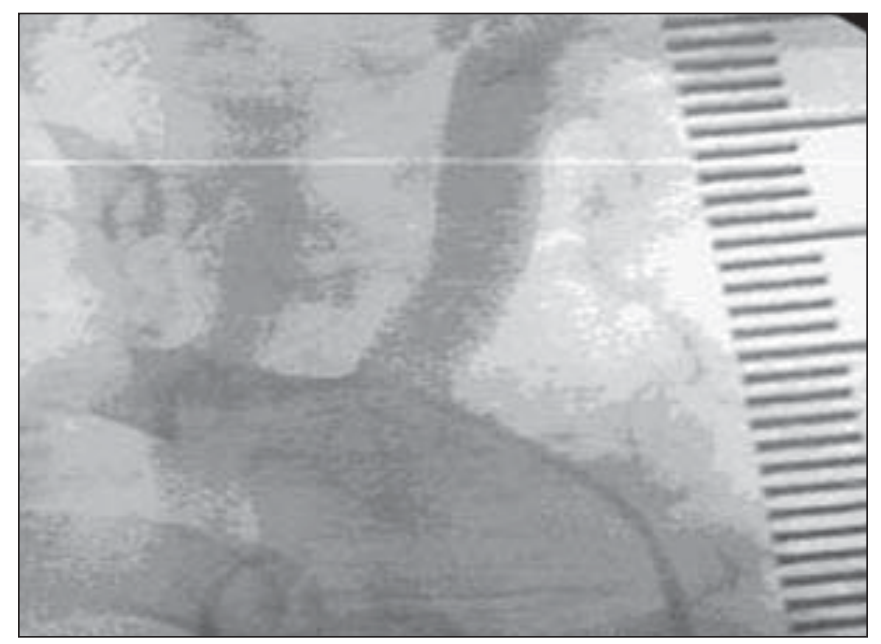

Figure 4: Final appearance after deployment of the stent with good recanalization and filling radial artery was palpable. She was offered radioactive iodine (I131) treatment or surgical treatment as treatment options for thyroid problem, both of which refused by her.

\section{Discussion}

Subclavian steal occurs when the subclavian artery becomes stenosed or occluded and blood flow is reversed in the ipsilateral vertebral artery because of the negative pressure gradient between the vertebro-basillary and vertebro-subclavian region. Subclavian steal is associated with a wide variety of signs and symptoms of vertebro-basilar insufficiency that include light-headedness, vertigo, motor deficits, focal seizures, confusion, upper extremity ischemia. ${ }^{[1-3]}$ Color Doppler sonography is a safe and noninvasive method to diagnose SSS. The vertebral flow in a patient with an increasing subclavian stenosis usually shows a gradual change from normal flow patterns, through intermediate stages to a complete flow reversal. ${ }^{[1-4]}$

Atherosclerosis is the common cause, in $95 \%$ of cases. Takayasu arteritis, neurofibromatosis, traumatic subclavian artery occlusion, hemodialysis fistula, aberrant subclavian artery and critical contralateral internal carotid artery stenosis can be recited among the other causes. ${ }^{[5-7]}$ In our case the stenosis was probably related to atherosclerosis. In subclavian artery stenosis, because of the numerous collaterals, ischemia of the extremity is uncommon. However provocative exercises may result in symptoms. ${ }^{[1,4]} \mathrm{In}$ our case, among records of 35 patients with SSS who experienced PTA-stent placement, she was the only patient with limb ischemia (left side). It appears that the left limb ischemia in our patient was probably related to stealing effect of the hypervascular thyroid nodule in the presence of subclavian stenosis. Clearance of limb ischemia following stent placement further support out hypothesis. Thyroid nodules are quite commonly seen, occurring in at least 4$7 \%$ of the population.

These nodules may be benign or malignant and may be very vascular. Radionucleotide thyroid angiography with technetium $99 \mathrm{~m}$ pertechnetate and power Doppler ultrasonography may be used to assess the vascularity of thyroid nodules. ${ }^{[2,8]}$ In our patient the thyroid particular nodule was highly vascular. This might have resulted in blood steal from the ipsilateral subclavian artery, which had already stolen from the contralateral vertebral artery via the vertebral artery. We could not find description of such a case in the literature written in English.

Treatment modalities of SSS include PTA with or without stenting and surgery. The reported success rates with PTA with or without stenting was between 74 and $100 \%{ }^{[4]}$ We performed direct stenting successfully to the stenosed subclavian artery in our patient.

In conclusion, if there are ischemic symptoms of the upper extremities in patients with subclavian artery stenosis, a concomitant hypervascular thyroid nodule should be investigated for possible stealing of the blood from the subclavian artery. 
Kanko et al: Subclavian steale with thyroid adenoma

\section{References}

1. Thomassen L, Aarli JA. Subclavian steal phenomenon. Clinical and hemodynamic aspects. Acta Neurol Scand 1994;90:241-4

2. Demirel K, Kapucu O, Yucel C, Ozdemir H, Avvaz G, Taneri F. A comparison of radionuclide thyroid angiography, $(99 \mathrm{~m})$ Tc-MIBI scintigraphy and power Doppler ultrasonography in the differential diagnosis of solitary cold thyroid nodules. Eur J Nucl Med Mol Imag 2003;30:642-50.

3. Bedi HS, Suri A, Kalkat MS, Sengar BS, Arora A, Sharma VP, et al. Subclavian steal syndrome associated with critical contralateral internal carotid artery stenosis: a hitherto unreported entity. Indian Heart .J 1999;51:310-2.

4. Kizilkilic O, Oguzkurt L, Terean F, Yalcin O, Tan M, Yildirim T. Subclavian Steal Syndrome from the Ipsilateral Vertebral Artery. Am J Neuroradiol 2004;25:1089-91
5. Roldan-Valadez E, Hernandez-Martinez P, Osorio-Peralta S, Elizalde-Acosta I Espinoza Cruz V, Casian-Castellanos G. Imaging diagnosis of subclavian steal syndrome secondary to Takayasu arteritis affecting a left-side subclavian artery. Arch Med Res 2003;34:433-8.

6. Omae T, Hirai Y, Fujjii K, Ikeda K, Ibayashi S, Iida M. Subclavian steal phenomenon induced by arteriovenous fistula for hemodialysis. Nippon Naika Gakkai Zasshi 2005;94:129-31.

7. Lee EB, Seo S. Acute symptomatic traumatic subclavian steal syndrome: case report. J Trauma 2003;55:370-1

8. Frates MC, Benson CB, Doubilet PM, Cibas ES, Marqusee E. Can color Doppler sonography aid in the prediction of malignancy of thyroid nodules? J Ultrasound Med 2003;22:127-31.

Accepted on 06-08-2005 